\title{
Protective effects of molecular hydrogen on steroid-induced osteonecrosis in rabbits via reducing oxidative stress and apoptosis
}

\author{
Jia Li ${ }^{1 *}$, Zhaogang $\mathrm{Ge}^{2}$, Lihong Fan ${ }^{3}$ and Kunzheng Wang ${ }^{3}$
}

\begin{abstract}
Background: The objective of this study was to investigate the protective effects of molecular hydrogen, a novel and selective antioxidant, on steroid-induced osteonecrosis $(\mathrm{ON})$ in a rabbit model.

Methods: Sixty rabbits were randomly divided into two groups (model group and hydrogen group). Osteonecrosis was induced according to an established protocol of steroid-induced ON. Rabbits in the hydrogen group were treated with intraperitoneal injections of molecular hydrogen at $10 \mathrm{ml} / \mathrm{kg}$ body weight for seven consecutive days. Plasma levels of total cholesterol, triglycerides, soluble thrombomodulin(STM), glutathione(GSH) and malondialdehyde(MDA) were measured before and after steroid administration. The presence or absence of ON was examined histopathologically. Oxidative injury and vascular injury were assessed in vivo by immunohistochemical staining of 8-hydoxy-2-deoxyguanosine(8-OHdG) and MDA, and ink artery infusion angiography. The terminal deoxynucleotidyl transferase-mediated dUTP nick end labeling (TUNEL) assays were performed to measure apoptosis.
\end{abstract}

Results: The incidence of steroid-induced ON was significantly lower in hydrogen group (28.6\%) than that in model group (68.0\%). No statistically differences were observed on the levels of total cholesterol and triglycerides.

Oxidative injury, vascular injury and apoptosis were attenuated in the hydrogen group compared with those in the model group in vivo.

Conclusions: These results suggested that molecular hydrogen prevents steroid-induced osteonecrosis in rabbits by suppressing oxidative injury, vascular injury and apoptosis.

Keywords: Molecular hydrogen, Osteonecrosis, Oxidative injury, Vascular injury, Apoptosis

\section{Background}

Steroid-induced osteonecrosis $(\mathrm{ON})$, especially at hip joint, is one of the most common complications of corticosteroids. As most of the affected patients are young individuals and the surgical prognosis of steroid-induced $\mathrm{ON}$ is generally poor, it is highly desirable to develop a strategy to prevent its occurrence. Hyperlipidemia, hypercoagulable condition, fat emboli, intraosseous pressure rise with fat cell enlargement, enhanced vasoconstriction and vascular endothelial dysfunction after steroid administration have been suggested to cause steroid-induced ON [1-3].

\footnotetext{
* Correspondence: happylee_xjtu@163.com

'Department of Orthopedics, The First Affiliated Hospital of Xi'an Jiaotong University, Yanta West Road, Xi'an, Shaanxi Province 710061, People's Republic of China

Full list of author information is available at the end of the article
}

However, the detailed pathophysiological mechanisms of steroid-induced $\mathrm{ON}$ remain unclear. It is generally accepted that microcirculatory disturbances within bone underlie the development of steroid-induced ON [4]. To clarify the pathophysiology of steroid-induced $\mathrm{ON}$ and develop promising methods to prevent its occurrence, a number of preclinical animal $\mathrm{ON}$ models have been established including traumatic $\mathrm{ON}$ (surgical vascular deprivation, physical and chemical insult-induced traumatic $\mathrm{ON}$ ) and non-traumatic $\mathrm{ON}$ (spontaneous, steroidinduced or steroid-associated, lipopolysaccharide-induced or their combination-induced, horse serum-induced and alcohol-induced) [5]. Many researchers described steroidinduced $\mathrm{ON}$ models in rabbits by administering methylprednisolone intramuscularly, which had an advantage 
with etiology similar to ON patients but also with disadvantages of only early-stage ON [6-8]. Multifocal ON lesions were identified histologically in the femora, implying the effectiveness of this model for the evaluation of therapeutic efficacy of interventions developed for prevention of steroid-induced $\mathrm{ON}$.

Recently, it was indicated that oxidative stress, which is associated with numerous pathological conditions including vascular injury and cell apoptosis $[9,10]$, plays a crucial role in the development of steroid-induced $\mathrm{ON}$ [11]. More recently, the pro-oxidant buthionine sulphoximine has been proved to successfully induce $\mathrm{ON}$ in rats model [12] and the antioxidative substances including vitamin $\mathrm{E}[13,14]$, glutathione (GSH) [11] and edaravone [7] have been demonstrated to be effective in preventing steroid-induced ON. Vascular injury and apoptosis, which can be induced by oxidative stress $[10,11]$, also participate in the development of steroid-induced ON $[15,16]$. Thus, we hypothesized that antioxidants may be effective in preventing the development of steroid-induced $\mathrm{ON}$ by blocking the development of oxidative stress, vascular injury and cell apoptosis.

In 2007, Ohsawa et al. [17] found that diatomic hydrogen $(\mathrm{H} 2)$ could selectively reduce cytotoxic reactive oxygen species(ROS) including hydroxyl radical $(\cdot \mathrm{OH})$ and peroxynitrite(ONOO-), without affecting physiological ROS including $\mathrm{H} 2 \mathrm{O} 2$ and the nitric oxide radical $(\mathrm{NO} \cdot)$, which have been shown to play important roles in immune defence systems and signal transduction [18-20]. Moreover, in a rat model of ischaemia-reperfusion brain injury, he and his colleagues observed that molecular hydrogen is more effective than edaravone and as effective as FK506 in alleviating oxidative injury, which indicated the potential of molecular hydrogen for therapy. Then, molecular hydrogen was proposed as a novel and selective antioxidant and researches on the potential use of hydrogen as therapeutic agents in various diseases were conducted. It was showed that hydrogen can exert beneficial effects in diverse diseases of animal models related to oxidative stress, including ischaemia-reperfusion injury, radiation complication, atherosclerosis and sepsis [21-24]. Besides, it was reported that administration of $\mathrm{H} 2$-saturated water can also improve lipid metabolism [25-27]. Compared with other antioxidants such as vitamin $\mathrm{E}$, vitamin $\mathrm{C}$ and edaravone, hydrogen gas can diffuse rapidly into tissue and subcellular compartments including mitochondria and the nucleus, and can further eliminate ROS generated in mitochondria and protect DNA from oxidative damage in nuclear [17]. Accordingly, we assumed that molecular hydrogen might exert beneficial effects on preventing steroid-induced $\mathrm{ON}$ by reducing oxidative stress and apoptosis. The aim of this study was to test whether hydrogen can prevent the development of steroid-induced $\mathrm{ON}$ and the possible mechanisms involved.

\section{Methods}

\section{Animal, grouping and treatment}

All the animals used were housed at the Experimental Animal Center of Xi'an Jiaotong University, China and were maintained on the standard laboratory diet and water ad libitum. All protocols were approved by the Animal Ethical Committee of the Xi'an Jiaotong University and were followed in accordance with the NIH Guide for the Care and Use of Laboratory Animals.

Sixty mature male New Zealand rabbits (age: 28 weeks) were divided into two groups (model group and hydrogen group), each containing 30 rabbits. Osteonecrosis was induced in both groups by methods according to the previously published protocols $[6,7]$. Briefly, the rabbits received an intramuscular injection of $20 \mathrm{mg} / \mathrm{kg}$ body weight of methylpednisolone acetate (MPSL, Pfizer Pharmaceutical, China) into the right gluteus medius muscle. In addition to MPSL, the hydrogen group also received intraperitoneal injections of molecular hydrogen at $10 \mathrm{ml} / \mathrm{kg}$ body weight for seven consecutive days starting from the day of MPSL administration, which was based on the period of greatest susceptibility to steroid-induced oxidative injury suggested by Ichiseki et al. [11, 28].

\section{Haematological examination}

The blood samples were obtained from the auricular artery of each rabbit in a fasting state immediately before injection of MPSL(day 0) and 3, 5, 7, 14 days after the MPSL injection. Blood samples were collected at the same time of the day. The levels of total cholesterol (T-cho; mg/dL), triglycerides (TG; mg/dL) and soluble thrombomodulin (sTM; ug/L) at day 0,7 , 14 and the level of $\mathrm{GSH}(\mathrm{ug} / \mathrm{mL})$ and $\mathrm{MDA}(\mathrm{umol} / \mathrm{L})$ at day $0,3,5,7,14$ were determined.

\section{Histopathology}

Two weeks after steroid administration, the rabbits were killed with an overdose of sodium pentobarbital. Bilateral femurs were obtained for histological analyses. For HE staining, bone samples were fixed with $10 \%$ neutral buffered formalin for 1 week, decalcified with $10 \%$ ethylene diamine tetraacetic acid (EDTA) for 4 weeks, then embedded in paraffin, cut into $4 \mu \mathrm{m}$ thick sections and stained with hematoxylin-eosin. The bone samples were cut along the coronal plane in the proximal one third and axial plane in the distal part (condyle).

Whole areas of bilateral femoral samples, including the proximal one-thirds and distal condyles, were histopathologically examined for the presence of $\mathrm{ON}$. A positive diagnosis of $\mathrm{ON}$ was determined based on the diffuse presence of empty lacunae or pyknotic nuclei of osteocytes within the bone trabeculae, accompanied by surrounding bone marrow cell necrosis or fat cell necrosis 
[29]. 8 sections were taken from the proximal one-thirds and distal condyles of both femurs of each rabbit. The presence or absence of $\mathrm{ON}$ was determined using well established criteria [29,30]. Briefly, rabbits that had at least one osteonecrotic lesion in the 8 sections were considered as $\mathrm{ON}+$, while those with no osteonecrotic lesions were defined as $\mathrm{ON}-$. Besides, the incidence of $\mathrm{ON}$ was calculated as the ratio of the number of rabbits with $\mathrm{ON} /$ total number of rabbits used in each group.

During the observation, the average bone marrow fat cell size was calculated using image analysis software (Image $1.32 \mathrm{j}, \mathrm{NIH}, \mathrm{USA}$ ). As described in previous reports, the average bone marrow fat cell size was defined as the average of the Feret's diameter of all bone marrow fat cells in 4 randomly-selected non-necrotic fields (16 fields for 4 dissected parts from each rabbit). Fat cells that had undergone necrosis were excluded from imaging analysis. During the observation, both the judgment of osteonecrosis and the calculation of average fat cell size were performed by two researchers who were blind to the identity of the hydrogen group.

\section{Ink artery infusion angiography}

Two weeks after steroid administration, the rabbits were anesthetized with sodium pentobarbital and ink artery infusion angiography was performed to investigate vascular injury in the femur according to the previously published protocol [31]. After the infusion, the animals were then euthanized and stored at $4{ }^{\circ} \mathrm{C}$ for $24 \mathrm{~h}$. Then the bilateral thighbones were dissected and the samples were fixed, decalcified, embedded and cut into $5-\mu \mathrm{m}$ thick slices. Ink leakage would be observed if vascular permeability increased which was induced by vascular injury.

\section{Immunohistochemistry}

To determine the level of oxidative injury, the femurs were stained immunohistochemically with anti-8-hydoxy-2deoxyguanosine (8-OHdG) monoclonal antibody (N45.1) and anti-malondialdehyde (MDA) monoclonal antibody (clone $1 \mathrm{~F} 83$ ) which was major marker of DNA oxidative injury and lipid peroxide respectively [14, 28]. Briefly, sections were immersed in 3\% hydrogen peroxide PBS for 10 min, rinsed several times in PBS, pretreated with $10 \%$ goat normal antiserum(Vector, Burlingame, California, USA) for $30 \mathrm{~min}$ at room temperature. Then, sections were reacted with a primary antibody overnight at $4{ }^{\circ} \mathrm{C}$ and incubated with the biotinylated secondary antibody for $30 \mathrm{~min}$, followed by streptavidin peroxidases for $30 \mathrm{~min}$ at room temperature. Finally, sections were treated with diaminobenzidine solution, counterstained with haematoxylin and mounted. Sections without primary antibodies processing were used as the negative control. The sections were observed and photographed by the eclipse $50 \mathrm{i}$ optical microscope imaging system (Nikon Co., Ltd., Tokyo, Japan).

To quantify DNA oxidation injury and vascular damage of immunohistological results, the 8-OHdG positive area per visual field (PA\%) and the MDA positive marrow vessels were calculated by image analysis software (ImageJ $1.32 \mathrm{j}, \mathrm{NIH}, \mathrm{USA}$ ). DNA oxidative injury was evaluated by calculating the \%PA of $8-\mathrm{OHdG}$, the percentage of 8 -OHdG positive area/total area, in 5 randomly selected visual fields ( $\times 200$ magnification) [7] Vascular damage was evaluated by counting the number of MDA positive marrow vessels in 5 randomly-selected fields from the proximal one-third of the femur $(\times 100$ magnification) [14].

\section{TUNEL assays}

The terminal deoxynucleotidyl transferase-mediated dUTP nick end labeling (TUNEL) assays were performed by using the TUNEL detection kit (Promega Co., Ltd., Beijing, China) to observe apoptotic osteoblasts and osteocytes according to the manufacturer's instructions. The cells with brown nuclei were assessed as positive. During the observation of per tissue slide at 200 times magnification, the apoptotic rate was calculated by two blinded researchers. The apoptotic rate was defined as number of TUNEL-positive cells/number of all cells. Besides, morphological changes characteristic of apoptosis were examined carefully to minimize ambiguity regarding the interpretation of positive results.

\section{Statistical analysis}

Categorical data, i.e. incidence of osteonecrosis, was analyzed using Pearson's chi-square test. All the numerical data were described as mean \pm standard deviation. Simple comparisons of numerical data were performed using Student's $t$-test. To compare the serial changes of GSH and MDA levels in each group, a repeatedmeasures analysis of variance (ANOVA) was used. To compare the changes in GSH and MDA values between the hydrogen group and the model group, a two-way ANOVA was used. The SPSS 17.0 software was used for analysis. $P$ values $<0.05$ were considered significant.

\section{Results}

\section{Histopathological observations}

Seven of the 60 rabbits ( 5 in the model group and 2 in the hydrogen group) died of the inductive protocol throughout the experiment period and were excluded from the evaluation.

Osteonecrosis was observed in 17 of the 25 rabbits in the model group and in 8 of 28 rabbits in the hydrogen group (Fig. 1). The incidence of osteonecrosis was significantly lower in the hydrogen group than that in the model group (Pearson's chi-square test, $P<0.01$ ). The size 

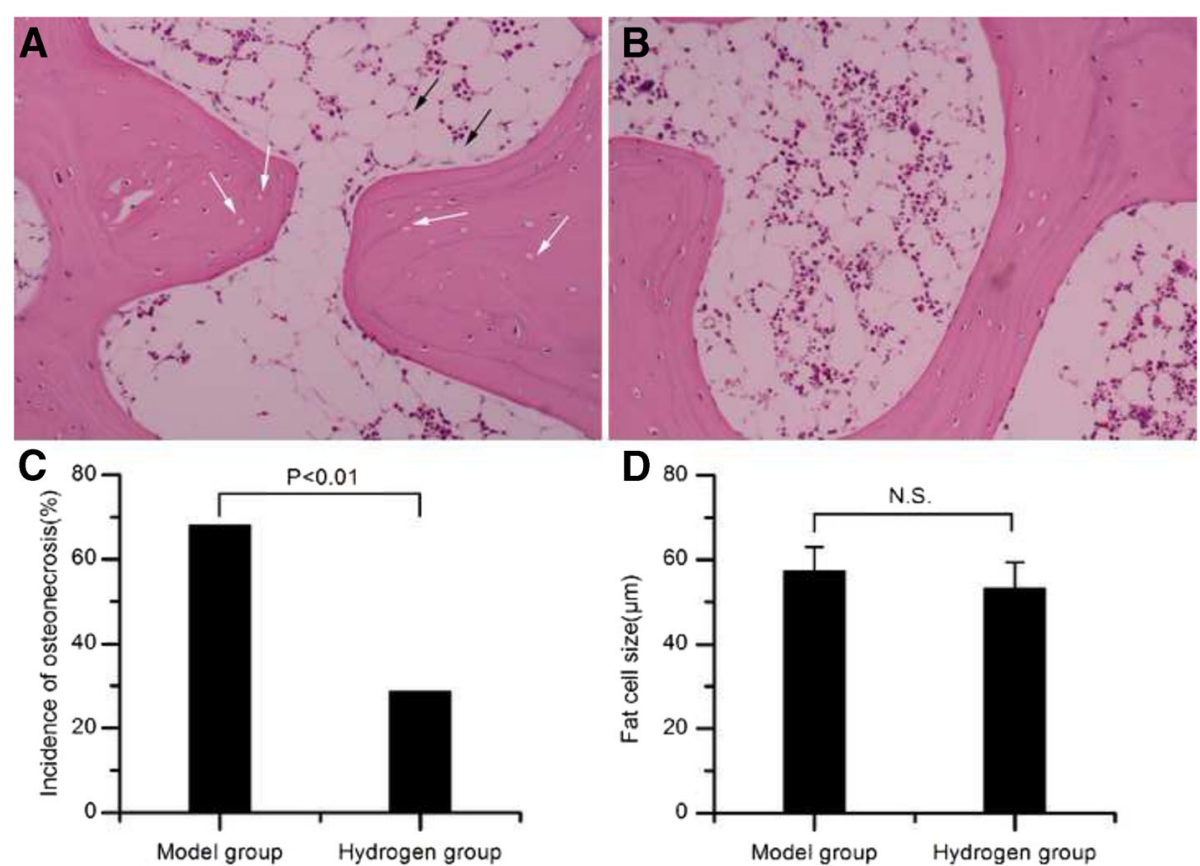

Fig. 1 Histological observation. a Representative ON+ sample in the model group showed more empty lacunae within the bone trabeculae (indicated by white arrow), surrounded by necrotic medullary haematopoietic cells and necrotic fat cells(indicated by black arrow). b Representative ON- sample in the hydrogen group showed less empty lacunae or osteocytes having pycnotic nuclei, surrounded by abundant normal marrow hematopoietic cells. $\mathbf{c}$ The ON incidence in the hydrogen group was significantly lower than that of the model group $(P<0.01)$. $\mathbf{d}$ There were no statistical difference about the size of fat cells between the hydrogen group and the model group $(P>0.05)$. N.S., not significant

of fat cells was $57.3 \pm 5.8 \mu \mathrm{m}$ in the model group and 53.6 $\pm 6.2 \mu \mathrm{m}$ in the hydrogen group $(P>0.05)$.

\section{Hematological findings}

Hematological data were compared both between different time points in the same group and between the model group and the hydrogen group at the same time points. The levels of total cholesterol and triglycerides in both groups increased during the period from day 7 to day 14 after MPSL administration $(P<0.05)$. However, there was no difference between the two groups at all the same time points $(P>0.05)$ (Fig. 2a and b). The sTM level significantly increased at day 7 in the model group and then declined toward baseline. However, in the hydrogen group, the significant increase of STM was prevented (Fig. 2c). In the model group, the GSH level was significantly decreased between 3 and 7 days after steroid administration $(P<0.05)$, followed by a tendency to recover by 7-14 days after administration. The MDA level was significantly increased between 5 and 7 days after steroid administration $(P<0.05)$ and appeared to recover by $7-14$ days after administration in the model group. Both decreases in GSH and increases in MDA of the hydrogen group were significantly attenuated compared with those in the model group (Fig. $2 \mathrm{~d}$ and e). In the hydrogen group, the decrease of GSH level was significantly suppressed on days 3 and 5 after MPSL administration $(P<0.05)$, and the increase of MDA level was significantly suppressed on day 7 after MPSL administration $(P<0.05)$.

Oxidative stress and vascular injury in the proximal femurs In marrow of the model group, clusters of 8-OHdGpositive haematopoietic cells were present (Fig. 3a). However, marrow of the hydrogen group showed only a few, sporadic 8-OHdG-positive haematopoietic cells (Fig. 3b). Furthermore, 8-OHdG \%PA was $2.3 \pm 0.5 \%$ in the hydrogen group (Fig. 3c), significantly lower than $5.4 \pm 0.8 \%$ in the model group $(P<0.01)$.

In the model group, strong immunoreactivity for MDA was found in marrow vessels and haematopoietic cells (Fig. 3d), whereas weak immunoreactivity for MDA was found in the hydrogen group (Fig. 3e). Furthermore, the number of MDA positive vessels was $4.1 \pm 1.9$ in the model group (Fig. 3f). In the hydrogen group, a smaller number of MDA positive vessels, $1.8 \pm 1.0$, was observed $(P<0.01)$.

Stronger ink leakage indicating increased vascular permeability was observed more often in the model group (Fig 3g). In the hydrogen group, less ink leakage was observed (Fig. 3h).

\section{Apoptosis in the proximal femurs}

In the model group, many TUNEL-positive osteoblasts and osteocytes were observed (Fig. 4a). However, few 


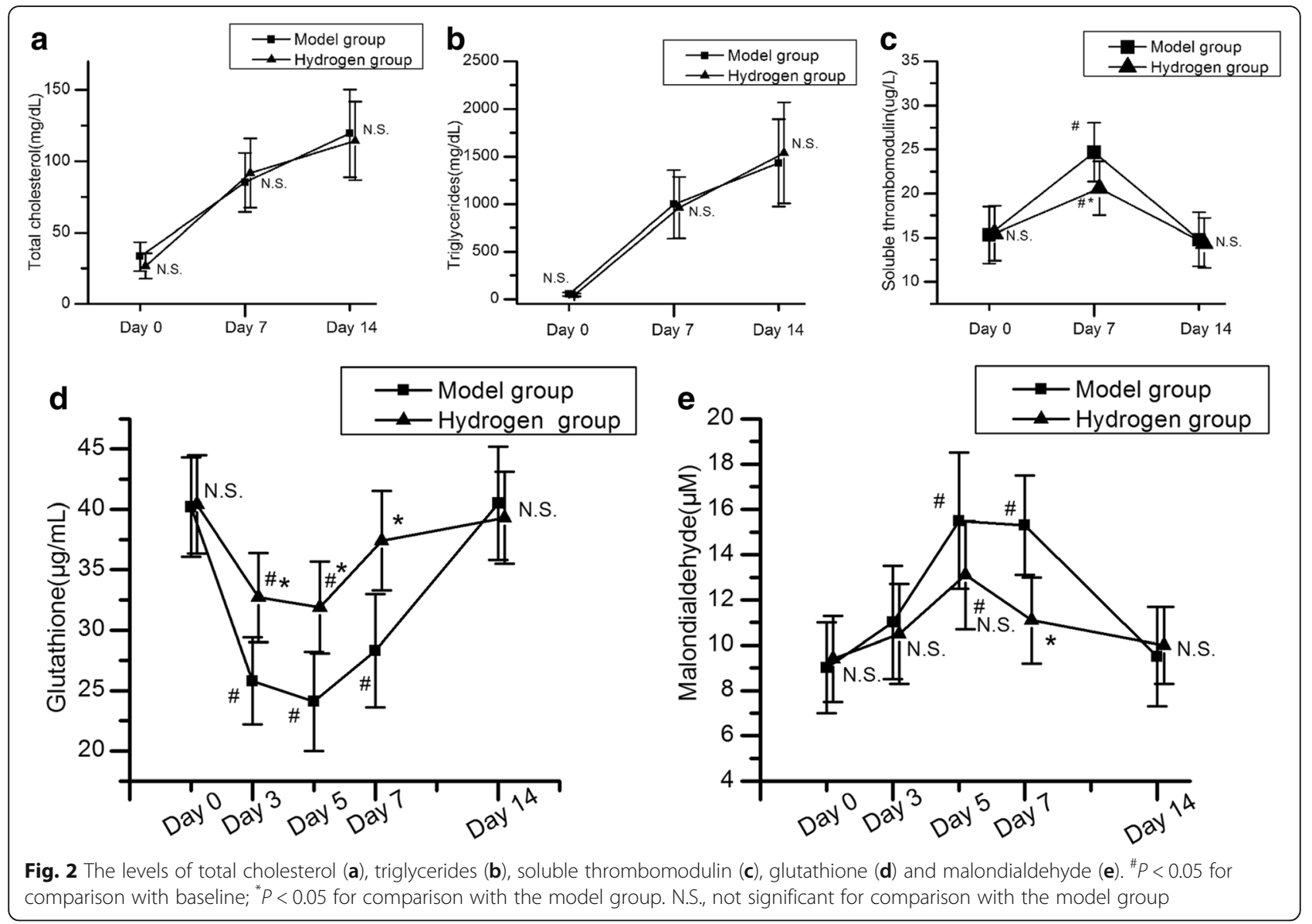

and sporadic TUNEL-positive osteoblasts and osteocytes were observed in hydrogen group (Fig. 4b), which indicated that the administration of molecular hydrogen protected the trabecular bone cells from apoptosis.

\section{Discussion}

Circulatory disturbance [32], vascular endothelial dysfunction [33], enhanced vasoconstriction [3], thrombotic and fibrinolytic disorders [2,34], impairment of lipid metabolism [35], increased oxidative stress [11, 28] and apoptosis [36] have been suggested to exert important roles in the development of steroid-induced osteonecrosis $(\mathrm{ON})$. Many drugs to inhibit these, including lipidlowering drugs (Statin) [6, 37], anticoagulants (Enoxaparin) $[2,38,39]$, antioxidants (Vitamin E, Lipoic acid, Edaravone) $[7,13,14,40]$ and vasodilation drugs [3] have been reported to effectively prevent or suppress the progression of steroid-induced $\mathrm{ON}$ in animals and humans. In the present study, we selected hydrogen gas, which was reported to decrease oxidative injury in various diseases related to oxidative stress and optimize lipid metabolism, as therapy for steroid-induced $\mathrm{ON}$ in an established rabbit model. We found that hydrogen therapy significantly decreased the incidence of steroid-induced $\mathrm{ON}$ in rabbits to $28 \%$ as compared with $68 \%$ in the model group, and attenuated both the decreases in plasma levels of GSH and the increases in plasma levels of MDA and sTM compared with those in the model group. Locally in the proximal femurs, DNA oxidative injury, lipid peroxide and vascular endothelial injury, indicated by immunohistochemistry for 8-OHdG, MDA and ink artery infusion angiography respectively, were decreased after hydrogen administration. However, effects of hydrogen on the levels of total cholesterol and triglycerides were not observed.

Disorders of lipid metabolism which resulted in extravascular lipid deposition and elevated intraosseous pressure, played an important role in the etiology of steroid-induced ON [35]. In the present study, disorders of lipid metabolism were evaluated by measuring the levels of total cholesterol and triglycerides as well as fat cell size. Total cholesterol, triglycerides and the fat cell size elevated after steroid administration which was consistent with findings by Kuribayashi et al. [14]. Study by Zong et al. [26] demonstrated that in high-fat diet-fed hamster model, 4-week intraperitoneal injection of hydrogen-saturated saline remarkably decreases plasma total cholesterol and low-density lipoprotein (LDL) cholesterol levels. In an open label, 8-week study on 20 

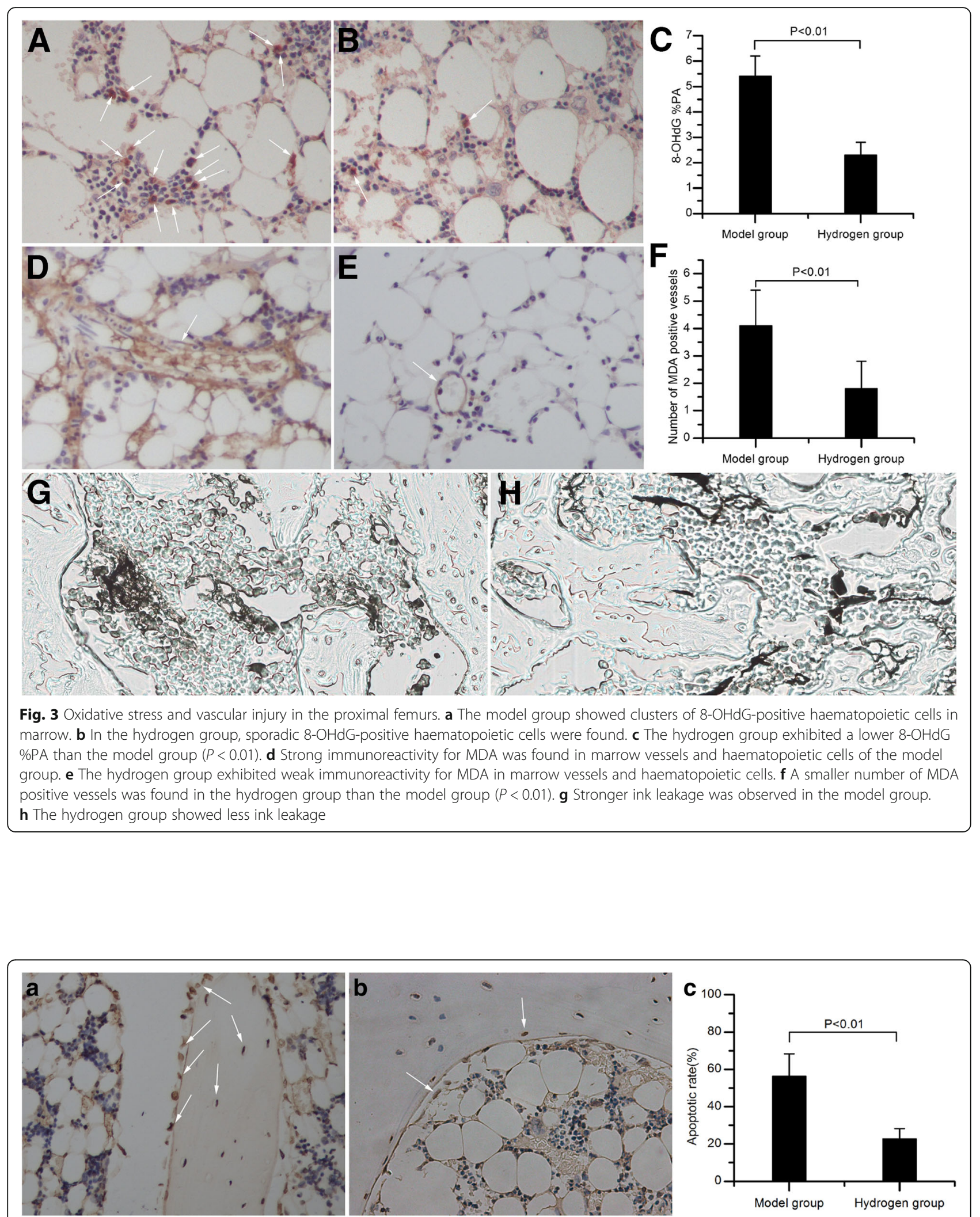

Fig. 4 TUNEL apoptosis detection. a The model group showed many apoptotic osteoblasts and osteocytes. $\mathbf{b}$ In the hydrogen group, few TUNEL-positive osteoblasts and osteocytes were found. $\mathbf{c}$ The apoptotic rate in the hydrogen group was significantly lower than that of the model group $(P<0.01)$ 
subjects with potential metabolic syndrome, Nakao et al. found administration of hydrogen rich water for 4 weeks increases high density lipoprotein (HDL)-cholesterol and decreases total cholesterol/high-density lipoprotein(HDL)cholesterol [25]. In addition, study of Kamimura et al. indicated that consuming molecular hydrogen for 3 months suppresses plasma triglyceride level in $\mathrm{db} / \mathrm{db}$ obesity model mice [27]. In our study, no statistically significant differences about the level of total cholesterol, triglycerides and the fat cell size were found between the model group and the hydrogen group, indicating that disorders of lipid metabolism was not improved by hydrogen during the 2-week duration for observation and some other osteonecrosis-prevention mechanisms exist other than disorders of lipid metabolism. Considering that the beneficial effects of molecular hydrogen on lipid metabolism were based on long-term molecular hydrogen administration, the period of hydrogen treatment and duration for observation should be extended to determine the longterm efficacy of hydrogen treatment on disorders of lipid metabolism in steroid-induced $\mathrm{ON}$.

Increased oxidative stress in the etiology of steroidinduced $\mathrm{ON}$ has been well documented $[7,11,13,14$, $28,40]$. In the present study, we measured the blood levels of GSH and MDA which generally reflect the development of oxidative stress in the body. GSH is an important antioxidant enzyme in vivo and exerts a crucial role in maintaining integrity of cell structure by inhibiting the increase of lipoperoxides and maintaining redox reaction balance. MDA is generated during the hyperoxidation of lipids and can be used as a biochemical indicator of oxidative injury. In our study, we found that GSH levels significantly decreased and MDA levels significantly increased shortly after MPSL administration. The results were in accord with those of Ichiseki et al. [11], Mikami et al. [13], Kuribayashi et al. [14] and Li et al. [7]. In addition, we detected the local expression of 8-OHdG and MDA, which was major marker of DNA oxidative injury and lipid peroxide respectively, in the femurs. Consistent with findings of previous studies $[7,14,28]$, the number of both 8-OHdG-positive haematopoietic cells and MDA-positive haematopoietic cells increased. In the prevention group treated with hydrogen, we found that the decrease of GSH and increase of MDA in plasma caused by steroid were significantly attenuated by hydrogen administration. Moreover, the number of both 8-OHdG-positive haematopoietic cells and MDApositive haematopoietic cells in marrow, and the 8-OHdG positive area per visual field (8-OHdG PA\%) decreased in group received hydrogen therapy. This demonstrated that hydrogen could systemically and locally reduce oxidative stress caused by steroid.

Vascular endothelial dysfunction injury was one of the factors that participate in the development of steroid- induced $\mathrm{ON}[33,41]$. It was reported that lipid peroxide could markedly damage vascular endothelial cells [42] and induce vascular endothelial dysfunction accompanying with oxidative injury in steroid-induced ON $[11,40]$. In our study, the results of immunohistochemical staining for MDA and ink artery infusion angiography in the group only received steroid indicated that bone marrow vessels suffered from extensive oxidative injury caused by steroid administration. However, hydrogen administration reduced the number of MDA-positive vessels in marrow compared with the model group, indicating that hydrogen considerably decreased the damage of steroid-induced oxidative stress to endothelial cells. Furthermore, we determined the level of soluble thrombomodulin(sTM) in plasma which is an index of endothelial injury. We found that sTM significantly increased in the model group at day 7 post induction and hydrogen reduced the sTM concentration.

Roles of apoptosis in steroid-induced ON were reported in several studies [15, 43]. Oxidative stress is an important contributor to apoptosis [10]. The results of TUNEL assay in our study demonstrated that molecular hydrogen decreased the number of apoptotic osteoblasts and osteocytes, which was in accord with the decrease of oxidative stress. It indicated that molecular hydrogen might indirectly inhibit apoptosis by suppressing oxidative stress.

Compared with conventional antioxidants, diatomic hydrogen has several potential advantages [17]. First, hydrogen is highly diffusible and it can penetrate biomembranes and diffuse into the mitochondria and nucleus, which are the primary site of ROS generation and DNA damage. Secondly, diatomic hydrogen $(\mathrm{H} 2)$ could selectively reduce level of hydroxyl radical $(\cdot \mathrm{OH})$ and peroxynitrite (ONOO-) which are the most aggressive ROS, but do not affect the level of $\mathrm{H} 2 \mathrm{O} 2$ and the nitric oxide radical $(\mathrm{NO} \bullet)$ which play physiological roles in immune defence systems and signal transduction. Thirdly, diatomic hydrogen exerts antioxidative effects with few toxic side effects at effective dosages. Finally, it is cheaper than conventional pharmaceuticals.

One of the limitations of the present study is that we limited the delivery of hydrogen gas to intraperitoneal injection and limited the therapeutic dose of hydrogen to $10 \mathrm{ml} / \mathrm{kg}$. We were unable to determine the optimum way of hydrogen administering and the optimum level of hydrogen. In addition, we did not clarify the further mechanisms at the gene level which might help us to better understand the preventive effects of hydrogen on osteonecrosis. However, it was indicated that genes for oxidoreduction-related proteins were up-regulated in the livers of rats after 4 weeks of drinking hydrogen-rich water [44]. This might partially clarify the antioxidative effects of hydrogen on osteonecrosis. Thus, in the near future, we will conduct additional studies to determine the optimum 
level of hydrogen that can prevent the development of osteonecrosis. Besides, we will determine the changes of oxidoreduction-related genes in the femurs after hydrogen administration.

\section{Conclusions}

In conclusion, the present results suggest that oxidative stress participated in the development of steroidinduced $\mathrm{ON}$ and molecular hydrogen could significantly reduce the incidence of steroid-induced ON possibly by systemically and locally reducing oxidative stress and endothelial injury. This might offer a novel and simple method to prevent the development of steroid-induced ON. Furthermore, the long-term efficacy of molecular hydrogen therapy on disorders of lipid metabolism in steroid-induced ON needs to be explored.

\section{Abbreviations \\ 8-OHdG: 8-hydoxy-2-deoxyguanosine; EDTA: Ethylene diamine tetraacetic acid; GSH: Glutathione; HDL: High density lipoprotein; HUVECs: Human umbilical vein endothelial cells; LDL: Low-density lipoprotein; \\ LPS: Lipopolysaccharide; MDA: Malondialdehyde; MPSL: Methylpednisolone acetate; ON: Osteonecrosis; ROS: Reactive oxygen species; sTM: Thrombomodulin; T-cho: Total cholesterol; TG: Triglycerides; TUNEL: The terminal deoxynucleotidyl transferase-mediated dUTP nick end labeling}

\section{Acknowledgements}

Not applicable.

\section{Funding}

This work was supported by the National Natural Science Foundation of China (Nos. 81101363, 81371944 and 81572145) and the Fundamental Research Funds for the Central Universities.

\section{Availability of data and materials}

The datasets supporting the conclusions of this article are included within the article and available from the corresponding author on reasonable request.

\section{Authors' contributions}

$J$ made substantial contributions to the conception and design of this manuscript, data acquisition, analysis and interpretation and the drafting of the manuscript. ZG carried out the haematological examination, immunohistochemistry and TUNEL analyses. LF participated in ink artery infusion angiography. KW participated in the design of the study and performed statistical analysis. All authors read and approved the final manuscript.

\section{Competing interests}

The authors declare that they have no competing interests.

\section{Consent for publication}

Not applicable.

\section{Ethics approval and consent to participate}

This study was carried out upon receiving approval by the Animal Ethical Committee of the Xi'an Jiaotong University was in strict accordance with the $\mathrm{NIH}$ Guide for the Care and Use of Laboratory Animals. All efforts were made to minimize suffering.

\section{Author details}

'Department of Orthopedics, The First Affiliated Hospital of Xi'an Jiaotong University, Yanta West Road, Xi'an, Shaanxi Province 710061, People's Republic of China. ${ }^{2}$ Department of Joint Surgery, Honghui Hospital of Xi'an Jiaotong University, Xi'an, Shaanxi Province 710054, People's Republic of China. ${ }^{3}$ The first department of Orthopedics, The Second Affiliated Hospital of Xi'an Jiaotong University, Xi'an, Shaanxi Province 710004, People's Republic of China.
Received: 9 June 2016 Accepted: 24 January 2017

Published online: 02 February 2017

\section{References}

1. Wang GJ, Cui Q, Balian G. The Nicolas Andry award. The pathogenesis and prevention of steroid-induced osteonecrosis. Clin Orthop Relat Res. 2000; (370):295-310

2. Beckmann $R$, Shaheen $H$, Kweider N, Ghassemi A, Fragoulis A, HermannsSachweh B, Pufe T, Kadyrov M, Drescher W. Enoxaparin prevents steroidrelated avascular necrosis of the femoral head. Scientific World J. 2014:2014:347813.

3. Drescher W, Beckmann R, Kasch R, Pufe M, Knobe M, Kweider N, Hassenpflug J, Tingart M, Pufe T, Kadyrov M. Nitrate patch prevents steroid-related bone necrosis. J orthop Sci. 2011;29(10):1517-20.

4. Mont MA, Hungerford DS. Non-traumatic avascular necrosis of the femoral head. J Bone Joint Surg Am. 1995;77(3):459-74.

5. Fan M, Peng J, Qin L, Lu S. Experimental animal models of osteonecrosis. Rheumatol Int. 2011;31(8):983-94.

6. Pengde $K$, Fuxing $P$, Bin S, Jing $Y$, Jingqiu C. Lovastatin inhibits adipogenesis and prevents osteonecrosis in steroid-treated rabbits. Joint Bone Spine. 2008;75(6):696-701.

7. Li GY, Feng Y, Cheng TS, Yin JM, Zhang CQ. Edaravone, a novel free radical scavenger, prevents steroid-induced osteonecrosis in rabbits. Rheumatol (Oxford, England). 2013:52(3):438-47.

8. Iwakiri K, Oda Y, Kaneshiro Y, Iwaki H, Masada T, Kobayashi A, Asada A, Takaoka K. Effect of simvastatin on steroid-induced osteonecrosis evidenced by the serum lipid level and hepatic cytochrome P4503A in a rabbit model. J Orthop Sci. 2008;13(5):463-8.

9. Baynes JW. Role of oxidative stress in development of complications in diabetes. Diabetes. 1991:40(4):405-12.

10. Hampton MB, Orrenius S. Redox regulation of apoptotic cell death. BioFactors (Oxford, England). 1998;8(1-2):1-5.

11. Ichiseki T, Matsumoto T, Nishino M, Kaneuji A, Katsuda S. Oxidative stress and vascular permeability in steroid-induced osteonecrosis model. J Orthop Sci. 2004:9(5):509-15.

12. Ichiseki T, Kaneuji A, Ueda Y, Nakagawa S, Mikami T, Fukui K, Matsumoto T. Osteonecrosis development in a novel rat model characterized by a single application of oxidative stress. Arthritis Rheum. 2011;63(7):2138-41.

13. Mikami T, Ichiseki T, Kaneuji A, Ueda Y, Sugimori T, Fukui K, Matsumoto T. Prevention of steroid-induced osteonecrosis by intravenous administration of vitamin E in a rabbit model. J Orthop Sci. 2010;15(5):674-7.

14. Kuribayashi M, Fujioka M, Takahashi KA, Arai Y, Ishida M, Goto T, Kubo T. Vitamin E prevents steroid-induced osteonecrosis in rabbits. Acta Orthop. 2010:81(1):154-60

15. Kabata T, Kubo T, Matsumoto T, Nishino M, Tomita K, Katsuda S, Horii T, Uto $\mathrm{N}$, Kitajima I. Apoptotic cell death in steroid induced osteonecrosis: an experimental study in rabbits. J Rheumatol. 2000;27(9):2166-71.

16. Zhang $G$, Qin $L$, Sheng $H$, Wang $X L$, Wang $Y X$, Yeung DK, Griffith JF, Yao XS, Xie XH, Li ZR, et al. A novel semisynthesized small molecule icaritin reduces incidence of steroid-associated osteonecrosis with inhibition of both thrombosis and lipid-deposition in a dose-dependent manner. Bone. 2009:44(2):345-56.

17. Ohsawa I, Ishikawa M, Takahashi K, Watanabe M, Nishimaki K, Yamagata K, Katsura K, Katayama Y, Asoh S, Ohta S. Hydrogen acts as a therapeutic antioxidant by selectively reducing cytotoxic oxygen radicals. Nat Med. 2007;13(6):688-94

18. Fang J, Seki $T$, Maeda $H$. Therapeutic strategies by modulating oxygen stress in cancer and inflammation. Adv Drug Deliv Rev. 2009;61(4):290-302.

19. Davies KJ. Oxidative stress: the paradox of aerobic life. Biochem Soc Symp. 1995;61:1-31

20. Sundaresan M, Yu ZX, Ferrans VJ, Irani K, Finkel T. Requirement for generation of $\mathrm{H} 2 \mathrm{O} 2$ for platelet-derived growth factor signal transduction. Sci (New York, NY). 1995;270(5234):296-9.

21. Cai J, Kang Z, Liu K, Liu W, Li R, Zhang JH, Luo X, Sun X. Neuroprotective effects of hydrogen saline in neonatal hypoxia-ischemia rat model. Brain Res. 2009:1256:129-37.

22. Qian L, Cao F, Cui J, Huang Y, Zhou X, Liu S, Cai J. Radioprotective effect of hydrogen in cultured cells and mice. Free Radic Res. 2010;44(3):275-82.

23. Ohsawa I, Nishimaki K, Yamagata K, Ishikawa M, Ohta S. Consumption of hydrogen water prevents atherosclerosis in apolipoprotein E knockout mice. Biochem Biophys Res Commun. 2008;377(4):1195-8. 
24. Xie K, Yu Y, Pei Y, Hou L, Chen S, Xiong L, Wang G. Protective effects of hydrogen gas on murine polymicrobial sepsis via reducing oxidative stress and HMGB1 release. Shock (Augusta, Ga). 2010;34(1):90-7.

25. Nakao A, Toyoda Y, Sharma P, Evans M, Guthrie N. Effectiveness of hydrogen rich water on antioxidant status of subjects with potential metabolic syndrome-an open label pilot study. J Clin Biochem Nutr. 2010;46(2):140-9.

26. Zong C, Song G, Yao S, Li L, Yu Y, Feng L, Guo S, Luo T, Qin S. Administration of hydrogen-saturated saline decreases plasma low-density lipoprotein cholesterol levels and improves high-density lipoprotein function in high-fat diet-fed hamsters. Metab Clin Exp. 2012;61(6):794-800.

27. Kamimura N, Nishimaki K, Ohsawa I, Ohta S. Molecular hydrogen improves obesity and diabetes by inducing hepatic FGF21 and stimulating energy metabolism in db/db mice. Obes (Silver Spring, Md). 2011;19(7):1396-403.

28. Ichiseki T, Kaneuji A, Katsuda S, Ueda Y, Sugimori T, Matsumoto T. DNA oxidation injury in bone early after steroid administration is involved in the pathogenesis of steroid-induced osteonecrosis. Rheumatol (Oxford, England). 2005;44(4):456-60.

29. Yamamoto T, Irisa T, Sugioka Y, Sueishi K. Effects of pulse methylprednisolone on bone and marrow tissues: corticosteroid-induced osteonecrosis in rabbits. Arthritis Rheum. 1997;40(11):2055-64.

30. Yamamoto T, Hirano K, Tsutsui H, Sugioka Y, Sueishi K. Corticosteroid enhances the experimental induction of osteonecrosis in rabbits with Shwartzman reaction. Clin Orthop Relat Res. 1995:316:235-43.

31. Fan L, Li J, Yu Z, Dang X, Wang K. Hypoxia-inducible factor prolyl hydroxylase inhibitor prevents steroid-associated osteonecrosis of the femoral head in rabbits by promoting angiogenesis and inhibiting apoptosis. PLoS One. 2014;9(9):e107774.

32. Powell C, Chang C, Gershwin ME. Current concepts on the pathogenesis and natural history of steroid-induced osteonecrosis. Clin Rev Allergy Immunol. 2011:41(1):102-13.

33. Boss $\mathrm{JH}$, Misselevich I. Osteonecrosis of the femoral head of laboratory animals: the lessons learned from a comparative study of osteonecrosis in man and experimental animals. Vet Pathol. 2003;40(4):345-54.

34. Glueck CJ, Freiberg RA, Fontaine RN, Tracy T, Wang P. Hypofibrinolysis, thrombophilia, osteonecrosis. Clin Orthop Relat Res. 2001;386:19-33.

35. Miyanishi K, Yamamoto T, Irisa T, Yamashita A, Jingushi S, Noguchi Y, Iwamoto Y. Bone marrow fat cell enlargement and a rise in intraosseous pressure in steroid-treated rabbits with osteonecrosis. Bone. 2002;30(1):185-90.

36. Calder JD, Buttery L, Revell PA, Pearse M, Polak JM. Apoptosis-a significant cause of bone cell death in osteonecrosis of the femoral head. J Bone Joint Surg British Volume. 2004;86(8):1209-13.

37. Pritchett JW. Statin therapy decreases the risk of osteonecrosis in patients receiving steroids. Clin Orthop Relat Res. 2001;386:173-8.

38. Glueck CJ, Freiberg RA, Sieve L, Wang P. Enoxaparin prevents progression of stages I and || osteonecrosis of the hip. Clin Orthop Relat Res. 2005;435:164-70.

39. Nagasawa K, Tada Y, Koarada S, Tsukamoto H, Horiuchi T, Yoshizawa S, Murai K, Ueda A, Haruta Y, Ohta A. Prevention of steroid-induced osteonecrosis of femoral head in systemic lupus erythematosus by anti-coagulant. Lupus. 2006;15(6):354-7.

40. Lu BB, Li KH. Lipoic acid prevents steroid-induced osteonecrosis in rabbits. Rheumatol Int. 2012;32(6):1679-83.

41. Kerachian MA, Harvey EJ, Cournoyer D, Chow TY, Seguin C. Avascular necrosis of the femoral head: vascular hypotheses. Endothelium. 2006;13(4):237-44.

42. Sasaguri Y, Nakashima T, Morimatsu M, Yagi K. Injury to cultured endothelial cells from human umbilical vein by linoleic acid hydroperoxide. J Appl Biochem. 1984;6(3):144-50.

43. Youm YS, Lee SY, Lee SH. Apoptosis in the osteonecrosis of the femoral head. Clin Orthop Surg. 2010;2(4):250-5.

44. Nakai Y, Sato B, Ushiama S, Okada S, Abe K, Arai S. Hepatic oxidoreductionrelated genes are upregulated by administration of hydrogen-saturated drinking water. Biosci Biotechnol Biochem. 2011;75(4):774-6.

\section{Submit your next manuscript to BioMed Central and we will help you at every step:}

- We accept pre-submission inquiries

- Our selector tool helps you to find the most relevant journal

- We provide round the clock customer support

- Convenient online submission

- Thorough peer review

- Inclusion in PubMed and all major indexing services

- Maximum visibility for your research

Submit your manuscript at www.biomedcentral.com/submit
Biomed Central 